\title{
Synthesis of Chiral Self-Assembling Rhombs and their Characterization in Solution, in the Gas- Phase, and at the Liquid-Solid Interface
}

\author{
Kyung Seok Jeong ${ }^{\S}$, Sun Young Kim ${ }^{\S}$, Ueon-Sang Shin ${ }^{\S}$, Michael Kogej ${ }^{\ddagger}$, Nguyen T. M. Hai ${ }^{\dagger}$,

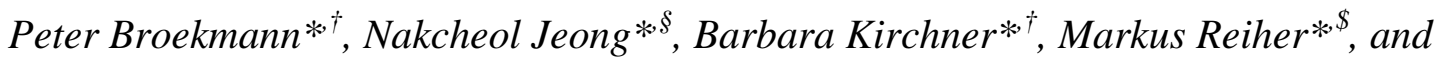 \\ Christoph A. Schalley ${ }^{*}$, \\ ${ }^{\S}$ Department of Chemistry and Division of Molecular Engineering and Chemistry, Korea \\ University \\ ${ }^{\dagger}$ Institut für Physikalische und Theoretische Chemie, Universität Bonn \\ ${ }^{\ddagger}$ Kekulé-Institut für Organische Chemie und Biochemie, Universtät Bonn \\ \$ Institut für Physikalische Chemie, Universität Jena
}

\section{Supporting Information}

\section{Synthetic Procedures and Analytical Data}

General Methods. Unless otherwise noted, all materials were obtained from commercial suppliers Aldrich and TCI, and used without further purification. Tetrahydrofuran (THF) was freshly distilled from sodium benzophenone ketyl prior to use. Toluene was distilled from calcium hydride and stored over $4 \AA$ molecular sieve. Organic solutions were concentrated using a Büchi rotary evaporator with a desktop vacuum pump. Thin layer chromatography plates (Silica gel $60 \mathrm{~F}_{254}$, Merck) were visualized by ultraviolet light and treatment with acidic p-anisaldehyde stain followed by gentle heating. Chromatographic purification of products were accomplished by flash chromatography, by using Merck silica gel 60 (230-400 mesh) with mixtures of hexane and ethyl acetate. ${ }^{1} \mathrm{H}$ NMR spectra were recorded on Varian Gemini $300 \mathrm{MHz}$. Chemical shifts are reported in ppm from TMS as internal standard $\left(\mathrm{CHCl}_{3}, \delta 7.26\right.$ ppm). ${ }^{13} \mathrm{C}$ NMR spectra were recorded on Varian Gemini $75 \mathrm{MHz}$ spectrometer with complete proton decoupling. Chemical shifts are reported in ppm from TMS as the internal 
reference $\left(\mathrm{CDCl}_{3}, \delta 77.7 \mathrm{ppm}\right)$. High Performance Liquid Chromatography (HPLC) was conducted using a Waters model 600 pumping system with a Waters model 2487 ultraviolet detector at $254 \mathrm{~nm}$. Infrared (IR) spectra were recorded on a Bomem 102 FT-IR spectrometer. Optical rotations were measured at the $589 \mathrm{~nm}$ sodium D-Line from RUDOLPH AUTOPOL automatic polarimeter. Low- and high-resolution EI mass spectra were obtained on a JEOL JMS-AX505WA mass spectrometer. Melting points were measured by Capillary melting point apparatus of Thomas Hoover and are uncorrected. The CD spectra were measured with a JASCO J810 spectropolarimeter. The samples were dissolved in 2,2,2-trifluoroethanol at a concentration of 30 $\mu \mathrm{M}$. The samples were measured at wavelengths of $180-400 \mathrm{~nm}$.

\section{Synthesis.}

Trans-4,4'-Dibromostilbene 4. To a stirred suspension of zinc powder (19.9 g, $302 \mathrm{mmol})$ in dry THF $(300 \mathrm{ml}), \mathrm{TiCl}_{4}(16.5 \mathrm{ml}, 151 \mathrm{mmol})$ was added slowly at $-10^{\circ} \mathrm{C}$. Then, a solution of 4-bromobenzaldehyde $(10.0 \mathrm{~g}, 54 \mathrm{mmol})$ in of dry THF $(250 \mathrm{ml})$ was added dropwise while the mixture was refluxed and stirred for $5 \mathrm{~h}$. The solution was quenched with saturated aqueous $\mathrm{NaHCO}_{3}$ solution and extracted with ethyl acetate (EA). The extract was washed with brine, dried on $\mathrm{MgSO}_{4}$, concentrated and washed with hexane (Hex) to give a white solid (8.84 g, 97 \%). $\mathrm{R}_{\mathrm{f}} 0.62$ (Hex : EA = $5: 1$ ); m.p. $=183-185^{\circ} \mathrm{C} ;{ }^{1} \mathrm{H} \mathrm{NMR}\left(\mathrm{CDCl}_{3}\right.$, $300 \mathrm{MHz}): \delta(\mathrm{ppm})=7.01(\mathrm{~s}, 2 \mathrm{H}), 7.37(\mathrm{~d}, 4 \mathrm{H}, \mathrm{J}=8.7 \mathrm{~Hz}), 7.49(\mathrm{~d}, 4 \mathrm{H}, \mathrm{J}=8.7 \mathrm{~Hz}) ;{ }^{13} \mathrm{C}$ NMR $\left(\mathrm{CDCl}_{3}, 75 \mathrm{MHz}\right): \delta(\mathrm{ppm})=121.62,127.99,128.10,131.84,135.88$; FT-IR $(\mathrm{KBr}$, $\mathrm{cm}^{-1}$ ) 3055; Anal. calcd. for $\mathrm{C}_{14} \mathrm{H}_{10} \mathrm{Br}_{2}$ : C: $49.74 \mathrm{H}: 2.98 \mathrm{Br}: 47.28$, found: C: $49.33 \mathrm{H}: 2.94$.

(S,S)-1,2-Bis(4-bromophenyl)-ethane-1,2-diol (S,S)-5. AD-mix- $\alpha$ (0.7 g, $1.4 \mathrm{~g}$ per mmol) was stirred in two phases of $t$-butyl alcohol $(2.5 \mathrm{ml})$ and water $(2.5 \mathrm{ml})$ at r.t. After the two phases were clearly formed, $\mathrm{CH}_{3} \mathrm{SO}_{2} \mathrm{NH}_{2}(48 \mathrm{mg}, 0.5 \mathrm{mmol})$ was added to the AD-mix- $\alpha$ solution at $0^{\circ} \mathrm{C}$. trans-4,4'-dibromostilbene $(120 \mathrm{mg}, 0.5 \mathrm{mmol})$ was added to the reaction mixture in one portion at $0^{\circ} \mathrm{C}$. The resultant reaction mixture was stirred at $0^{\circ} \mathrm{C}$ for $20 \mathrm{~h}$. After completion of the reaction, the mixture was quenched with a saturated aqueous $\mathrm{Na}_{2} \mathrm{CO}_{3}$ solution and extracted with ethyl acetate (EA) three times. The combined extract was washed with brine, dried on anhydrous $\mathrm{MgSO}_{4}$ and filtered. The filtrate was concentrated under reduced pressure to give a pale yellow precipitate. The crude product was purified by column chromatography $\left(\mathrm{SiO}_{2}\right)$ with an eluent of $\mathrm{Hex} / \mathrm{EA}=1: 1$. A white solid $(103 \mathrm{mg})$ was obtained in $75 \%$ yield. $\mathrm{R}_{\mathrm{f}} 0.45(\mathrm{Hex}: \mathrm{EA}=1: 1) ; \mathrm{m} . \mathrm{p} .=112^{\circ} \mathrm{C} ;{ }^{1} \mathrm{H} \mathrm{NMR}\left(\mathrm{CDCl}_{3}, 300 \mathrm{MHz}\right): \delta(\mathrm{ppm})$ $=3.27(\mathrm{~s}, 2 \mathrm{H}), 4.48(\mathrm{~s}, 2 \mathrm{H}), 6.90(\mathrm{~d}, 4 \mathrm{H}, \mathrm{J}=8.4 \mathrm{~Hz}), 7.34(\mathrm{~d}, 4 \mathrm{H}, \mathrm{J}=8.4 \mathrm{~Hz}) .{ }^{13} \mathrm{C} \mathrm{NMR}$ 
$\left(\mathrm{CDCl}_{3}, 75 \mathrm{MHz}\right): \delta(\mathrm{ppm})=78.42,122.02,128.62,131.31,138.38$; FT-IR $\left(\mathrm{KBr}, \mathrm{cm}^{-1}\right) 3379$, 3055, 2933; negative ESI-FTICR MS (methanol): $\mathrm{m} / \mathrm{z}=370.91(100 \%)\left[\mathrm{MH}^{+}\right], 742.81(15 \%)$ $\left[\mathrm{M}_{2} \mathrm{H}^{+}\right]$; Anal. calcd. for $\mathrm{C}_{14} \mathrm{H}_{12} \mathrm{Br}_{2} \mathrm{O}_{2}$ : C: $45.20 \mathrm{H}: 3.25 \mathrm{Br}: 42.95 \mathrm{O}: 8.60$, found $\mathrm{C}: 45.14 \mathrm{H}$ : 3.23; HPLC (Daicel Chiralpak AD-H, n-Hex/IPA = $9: 1$, flow $0.5 \mathrm{ml} / \mathrm{min}$, detection at 254 $\mathrm{nm}): \tau_{\mathrm{R}}=20.25 \mathrm{~min} . ;[\alpha]_{\mathrm{D}}{ }^{18}=-1434^{\circ}\left(\mathrm{C}=0.0052 \mathrm{~g} / \mathrm{ml}, \mathrm{CHCl}_{3}, 99.9 \%\right.$ ee $)$.

$(R, R)-1,2-B i s(4-b r o m o p h e n y l)-e t h a n e-1,2-d i o l$ (R,R)-5. Same procedure as above with $5.00 \mathrm{~g}$ of trans-4,4'-dibromostilbene (75\% yield), but using AD-mix- $\beta$ instead of AD-mix$\alpha$, provided $4.12 \mathrm{~g}$ of $(\mathrm{R}, \mathrm{R})-5$ (75\% yield). Spectral data identical with those of $(S, S)-5$ except for $[\alpha]_{\mathrm{D}}{ }^{18}=+1418^{\circ}\left(\mathrm{C}=0.0052 \mathrm{~g} / \mathrm{ml} \mathrm{CHCl}_{3}, 99.8 \%\right.$ ee $)$.

(S,S)-1,2-Bis(4-bromophenyl)-1,2-dimethoxy-ethane (S,S)-6. A solution of (S,S)-1,2-bis(4bromophenyl)-ethane-1,2-diol (1.00 g, $2.69 \mathrm{mmol})$ was slowly added to a suspension of $\mathrm{NaH}$ $(60 \%$ in oil, $533 \mathrm{mg}, 13.44 \mathrm{mmol})$ in dry THF $(20 \mathrm{ml})$ at $0^{\circ} \mathrm{C}$ under argon atmosphere. After completion of gas evolution after ca. $10 \mathrm{~min}$, a solution of iodomethane $(0.58 \mathrm{ml}, 9.4 \mathrm{mmol})$ in THF $(1 \mathrm{~mL})$ was introduced into the reaction mixture at r.t. After stirring of the resulting reaction mixture for $24 \mathrm{~h}$, the reaction was quenched with water carefully and partitioned between water and ethyl acetate. The organic extracts were combined and washed with brine, dried over anhydrous $\mathrm{MgSO}_{4}$ and filtered. The filtrate was concentrated under reduced pressure to give a white solid. The crude product was purified by column chromatography $\left(\mathrm{SiO}_{2}\right.$, eluent: Hex : EA = 5:1) to afford a white solid (1.08 g, $\left.100 \%\right) . \mathrm{R}_{\mathrm{f}} 0.43(\mathrm{Hex}: \mathrm{EA}=5$ : 1); m.p. $=73{ }^{\circ} \mathrm{C} ;{ }^{1} \mathrm{H} \mathrm{NMR}\left(\mathrm{CDCl}_{3}, 300 \mathrm{MHz}\right): \delta(\mathrm{ppm})=3.23(\mathrm{~s}, 6 \mathrm{H}), 4.24(\mathrm{~s}, 2 \mathrm{H}), 6.87(\mathrm{~d}$, $4 \mathrm{H}, \mathrm{J}=8.4 \mathrm{~Hz}), 7.33(\mathrm{~d}, 4 \mathrm{H}, \mathrm{J}=8.4 \mathrm{~Hz}) ;{ }^{13} \mathrm{C} \mathrm{NMR}\left(\mathrm{CDCl}_{3}, 75 \mathrm{MHz}\right): \delta(\mathrm{ppm})=57.25$, 86.44, 121.76, 129.50, 131.10, 136.88; FT-IR (KBr, cm $\left.{ }^{-1}\right): 3059,2916$; MS (EI, $\left.70 \mathrm{ev}\right): \mathrm{m} / \mathrm{z}=$ $397.8(0.1 \%)\left[\mathrm{M}^{+\bullet}\right], 366.8(0.6 \%)\left[\mathrm{M}-\mathrm{OCH}_{3}\right]^{+}, 335.9(0.5 \%)\left[\mathrm{M}-2 \mathrm{OCH}_{3}\right]^{+\bullet}, 318.8(0.1 \%)[\mathrm{M}-$

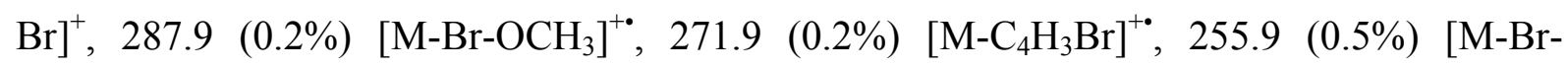
$\left.2 \mathrm{OCH}_{3}\right]^{+}, 198.9(100 \%)\left[\mathrm{M}-\mathrm{CH}_{3} \mathrm{OCH}-\mathrm{C}_{6} \mathrm{H}_{4} \mathrm{Br}\right]^{+}$(isotope patterns are in agreement with these assignments of parent and fragments). Anal. calcd. for $\mathrm{C}_{16} \mathrm{H}_{16} \mathrm{Br}_{2} \mathrm{O}_{2}$ : C: $48.03 \mathrm{H}: 4.03 \mathrm{Br}$ : 39.94 O: 8.00 , found: $\mathrm{C}: 48.13 \mathrm{H}: 4.22 ;[\alpha]_{\mathrm{D}}{ }^{17}=-153^{\circ}\left(\mathrm{C}=0.005 \mathrm{~g} / \mathrm{ml}, \mathrm{CHCl}_{3}\right)$.

(R,R)-1,2-Bis(4-bromophenyl)-1,2-dimethoxy-ethane (R,R)-6. Same procedure as above with $2.00 \mathrm{~g}(5.37 \mathrm{mmol})$ of $(R, R)-5$ provided $2.13 \mathrm{~g}(5.32 \mathrm{mmol} ; 99 \%)$ of $(R, R)-6$ as a white solid. Spectral data identical with those of $(S, S)-6$ except for $[\alpha]_{\mathrm{D}}{ }^{17}=+153^{\circ}(\mathrm{C}=0.005 \mathrm{~g} / \mathrm{ml}$, $\left.\mathrm{CHCl}_{3}\right)$. 
(S,S)-1,2-Bis(4-dihydroxyboranyl-phenyl)-1,2-dimethoxy-ethane (S,S)-7. A solution of $n$ butyllithium (15.2 $\mathrm{ml}$ of 1.6 M solution in hexane, $4.00 \mathrm{mmol}$ ) was added slowly to a solution of $(S, S)$-1,2-bis(4-bromopheny)-1,2-dimethoxy-ethane $(4.00 \mathrm{~g}, 10.0 \mathrm{mmol})$ in dry THF (50 $\mathrm{ml}$ ) at $-78^{\circ} \mathrm{C}$ under argon atmosphere. After stirring for $30 \mathrm{~min}$. at $-78^{\circ} \mathrm{C}$, trimethyl borate $(8.8 \mathrm{ml}, 80 \mathrm{mmol})$ was added, and the reaction mixture was allowed to warm to r.t. while stirring for $12 \mathrm{~h}$. The reaction was quenched with an aqueous solution of $\mathrm{HCl}(1 \mathrm{M})$ and extracted with ethyl acetate. The crude organic extract was treated with an aqueous $\mathrm{NaOH}$ solution $(1 \mathrm{M}, 100 \mathrm{~mL})$ three times. The combined aqueous extracts were acidified with conc. $\mathrm{HCl}$ and extracted with ethyl acetate. The organic extracts were combined and washed with brine, dried on anhydrous $\mathrm{MgSO}_{4}$ and filtered. The filtrate was then concentrated under reduced pressure to give a solid crude product. The residue was purified by column chromatography $\left(\mathrm{SiO}_{2}\right.$, with eluent dichloromethane $: \mathrm{MeOH}=10: 1$ to provide the product as a white solid (2.45 g, $74 \%) . \mathrm{R}_{\mathrm{f}} 0.35(\mathrm{MC}: \mathrm{MeOH}=10: 1)$; m.p. $=83-85^{\circ} \mathrm{C} ;{ }^{1} \mathrm{H} \mathrm{NMR}$ $\left(\mathrm{CD}_{3} \mathrm{OD}, 300 \mathrm{MHz}\right): \delta(\mathrm{ppm})=3.17(\mathrm{~s}, 6 \mathrm{H}), 4.27(\mathrm{~s}, 2 \mathrm{H}), 6.92(\mathrm{~d}, 4 \mathrm{H}, \mathrm{J}=7.7 \mathrm{~Hz}), 7.47(\mathrm{~d}$, $4 \mathrm{H}, \mathrm{J}=7.7 \mathrm{~Hz}) ;{ }^{13} \mathrm{C}$ NMR $\left(\left[\mathrm{D}_{6}\right]-\mathrm{DMSO}, 75 \mathrm{MHz}\right): \delta(\mathrm{ppm})=56.56,86.45,126.95,133.54$, 140.17; FT-IR (KBr, $\mathrm{cm}^{-1}$ ) 3393, 2929; negative ESI-FTICR MS (methanol): $\mathrm{m} / \mathrm{z}=329.14$ $\left[\mathrm{M}-\mathrm{H}^{+}\right]$, in addition signals for an exchange of the hydroxy groups against methoxy are observed as well as cyclic dimers and trimers formed through condensation.

$(R, R)-1,2-B i s(4-d i h y d r o x y b o r a n y l-p h e n y l)-1,2-d i m e t h o x y-e t h a n e(S, S)-7$. Same procedure as above with $2.00 \mathrm{~g}(4.99 \mathrm{mmol})$ of $(R, R)-6$ provided $1.18 \mathrm{~g}(3.60 \mathrm{mmol} ; 72 \%)$ of $(R, R)-7$ as a white solid. Spectral data identical with those of $(S, S)-7$.

(S,S)-1,2-Bis(4-(4-pyridyl)-phenyl)-1,2-dimethoxy-ethane (S,S)-8. 4-Bromopyridine hydrochloride (1.18 g, $6.06 \mathrm{mmol})$ was added in one portion to a mixture of (S,S)-1,2-bis(4dihydroxyboranyl-pheny)-1,2-dimethoxy-ethane (1.00 g, $3.03 \mathrm{mmol}), \mathrm{Pd}\left(\mathrm{PPh}_{3}\right)_{4}(350 \mathrm{mg}$, $0.303 \mathrm{mmol})$ and $\mathrm{Na}_{2} \mathrm{CO}_{3}(11.4 \mathrm{~g}, 108 \mathrm{mmol})$ in toluene $(72 \mathrm{ml})$ and water $(54 \mathrm{ml})$. The reaction mixture was heated at $120^{\circ} \mathrm{C}$ for $24 \mathrm{~h}$. After completion of the reaction, the mixture was quenched with aqueous $\mathrm{NaOH}$ solution and extracted with dichloromethane. The organic extracts were combined and washed with brine, dried on anhydrous $\mathrm{MgSO}_{4}$ and filtered. The filtrate was then concentrated under reduced pressure to give a solid crude product. The residue was purified by column chromatography $\left(\mathrm{SiO}_{2}\right.$, with eluent of hexane : ethyl acetate $=$ $1: 3)$ to provide the product as a white solid $(690 \mathrm{mg}, 87 \%) . \mathrm{R}_{\mathrm{f}} 0.14(\mathrm{Hex}: \mathrm{EA}=1: 3)$; m.p. 
$=220^{\circ} \mathrm{C} ;{ }^{1} \mathrm{H} \mathrm{NMR}\left(\mathrm{CDCl}_{3}, 300 \mathrm{MHz}\right): \delta(\mathrm{ppm})=3.31(\mathrm{~s}, 6 \mathrm{H}), 4.42(\mathrm{~s}, 2 \mathrm{H}), 7.18(\mathrm{~d}, 4 \mathrm{H}, \mathrm{J}=$ $8.0 \mathrm{~Hz}), 7.50(\mathrm{~m}, 8 \mathrm{H}) 8.63(\mathrm{~d}, 4 \mathrm{H}, \mathrm{J}=4.4 \mathrm{~Hz}) ;{ }^{13} \mathrm{C} \mathrm{NMR}\left(\mathrm{CDCl}_{3}, 75 \mathrm{MHz}\right): \delta(\mathrm{ppm})=57.7$, 87.1, 121.7, 126.8, 128.9, 137.7, 139.5, 148.0, 150.5; FT-IR $\left(\mathrm{KBr}, \mathrm{cm}^{-1}\right)$ 3077, 2938; positive ESI-FTICR MS (methanol): $\mathrm{m} / \mathrm{z}=397.19(20 \%)\left[\mathrm{MH}^{+}\right], 419.18(100 \%)\left[\mathrm{MNa}^{+}\right] ;[\alpha]_{\mathrm{D}}{ }^{17}=$ $-647^{\circ}\left(\mathrm{C}=0.0051 \mathrm{~g} / \mathrm{ml}, \mathrm{CHCl}_{3}\right)$.

(R,R)-1,2-Bis(4-(4-pyridyl)-phenyl)-1,2-dimethoxy-ethane $(R, R)-8$. Same procedure as above with $1.00 \mathrm{~g}(3.03 \mathrm{mmol})$ of $(R, R)-7$ provided $0.95 \mathrm{~g}(2.39 \mathrm{mmol} ; 85 \%)$ of $(R, R)-8$ as a white solid. Spectral data identical with those of $(S, S)-8$ except for $[\alpha]_{D}{ }^{17}=+647^{\circ}(\mathrm{C}=$ $\left.0.0051, \mathrm{CHCl}_{3}\right)$.

(S,S)-4,5-bis-(4-bromophenyl)-2,2-dimethyl-[1,3]dioxolane (S,S)-9. A mixture of (S,S)-1,2bis(4-bromophenyl)-ethane-1,2-diol (S,S)-5 (15.60 g, $42 \mathrm{mmol})$, p-TsOH $\bullet \mathrm{H}_{2} \mathrm{O}$ (319 mg, 1.68 mmol, $4 \mathrm{~mol} \%$ ), 2,2-dimethoxypropane (7.7 ml, $62.9 \mathrm{mmol})$, cyclohexane (600 $\mathrm{ml}, 0.07 \mathrm{M})$ was stirred under reflux with a Dean-Stark trap. After $3 \mathrm{~h}$, the reaction mixture was concentrated in vacuo to give crude product as a dark oil. This was purified by column chromatograpy $\left(\mathrm{SiO}_{2}\right.$, eluent: Hex : $\left.\mathrm{EA}=5: 1\right)$ to afford a white solid $(16.00 \mathrm{~g}, 92 \%)$. $\mathrm{R}_{\mathrm{f}}$ 0.65 (Hex : EA = $5: 1)$; m.p. $=130{ }^{\circ} \mathrm{C} ;{ }^{1} \mathrm{H}$ NMR $\left(\mathrm{CDCl}_{3}, 300 \mathrm{MHz}\right): \delta(\mathrm{ppm})=1.66(\mathrm{~s}, 6 \mathrm{H})$, $4.62(\mathrm{~s}, 2 \mathrm{H}), 7.08(\mathrm{~d}, 4 \mathrm{H}, \mathrm{J}=8.4 \mathrm{~Hz}), 7.46(\mathrm{~d}, 4 \mathrm{H}, \mathrm{J}=8.4 \mathrm{~Hz}) ;{ }^{13} \mathrm{C} \mathrm{NMR}\left(\mathrm{CDCl}_{3}, 75 \mathrm{MHz}\right): \delta$ $(\mathrm{ppm})=27.08,84.78,109.76,122.33,128.24,131.64,135.40 ; \mathrm{FT}-\mathrm{IR}\left(\mathrm{KBr}, \mathrm{cm}^{-1}\right): 2996$, 2884, 1911, 1601 ; Anal. calcd. for $\mathrm{C}_{16} \mathrm{H}_{16} \mathrm{Br}_{2} \mathrm{O}_{2}$ : C: $49.54 \mathrm{H}: 3.91 \mathrm{Br}: 38.78 \mathrm{O}: 7.76$, found: $\mathrm{C}: 49.53192 \mathrm{H}: 3.90078 ;[\alpha]_{\mathrm{D}}^{20}=-1329^{\circ}\left(\mathrm{C}=0.0425 \mathrm{~g} / \mathrm{L}, \mathrm{CHCl}_{3}\right)$.

(S,S)-4,5-bis-(4-dihydroxyboranyl-phenyl)-2,2-dimethyl-[1,3]dioxolane (S,S)-10. A solution of $n$-butyllithium (11.65 $\mathrm{ml}$ of $2.5 \mathrm{M}$ solution in hexane, $29.11 \mathrm{mmol}$ ) was added slowly to a solution of (S,S)-4,5-bis-(4-bromo phenyl)-2,2-dimethyl-[1,3]dioxolane (S,S)-9 (6.00 g, 14.56 $\mathrm{mmol})$ in dry THF $(145 \mathrm{ml})$ at $-78^{\circ} \mathrm{C}$ under argon atmosphere. After stirring for $30 \mathrm{~min}$. at $78^{\circ} \mathrm{C}$, trimethyl borate $(3.5 \mathrm{ml}, 30.58 \mathrm{mmol})$ was added, and the reaction mixture was allowed to warm to r.t. while stirring for $12 \mathrm{~h}$. The reaction was quenched with an aqueous solution of $\mathrm{HCl}(1 \mathrm{M})$ and extracted with ethyl acetate. The crude organic extract was treated with an aqueous $\mathrm{NaOH}$ solution $(1 \mathrm{M}, 50 \mathrm{ml})$ three times. The combined aqueous extracts were acidified with $\mathrm{HCl}(1 \mathrm{M}, 160 \mathrm{ml})$ and extracted with ethyl acetate. The organic extracts were combined and washed with brine, dried on anhydrous $\mathrm{MgSO}_{4}$ and filtered. The filtrate was then concentrated under reduced pressure to give a solid crude product. The residue was 
purified by recrystalization to provide the product as a white solid (3.34 g, 67\%). $\mathrm{R}_{\mathrm{f}} 0.5$ (MC $: \mathrm{MeOH}=10: 1)$; m.p. $=259 \sim 261{ }^{\circ} \mathrm{C} ;{ }^{1} \mathrm{H}$ NMR $\left(\left[\mathrm{D}_{6}\right]-\mathrm{DMSO}, 300 \mathrm{MHz}\right): \delta(\mathrm{ppm})=1.58(\mathrm{~s}$, $6 \mathrm{H}), 4.74(\mathrm{~s}, 2 \mathrm{H}), 7.17(\mathrm{~d}, 4 \mathrm{H}, \mathrm{J}=7.6 \mathrm{~Hz}), 7.74$ (d, 4H, J = $7.4 \mathrm{~Hz}), 8.06$ (s, 4H); ${ }^{13} \mathrm{C} \mathrm{NMR}$ $\left(\left[\mathrm{D}_{6}\right]-\mathrm{DMSO}, 75 \mathrm{MHz}\right): \delta(\mathrm{ppm})=27.74,85.16,109.50,126.61,134.77,138.95 ;$ FT-IR $(\mathrm{KBr}$, $\left.\mathrm{cm}^{-1}\right): 3426,2991,1603 ;[\alpha]_{\mathrm{D}}^{20}=-1552^{\circ}\left(\mathrm{C}=0.0113 \mathrm{~g} / \mathrm{L}, \mathrm{CH}_{3} \mathrm{OH}\right)$

(S,S)-4,5-Bis-(4-(4-pyridyl)-phenyl)-2,2-dimethyl-[1,3]dioxolane (S,S)-11. 4-Bromopyridine hydrochloride (2.40 g, $12.8 \mathrm{mmol})$ was added in one portion to a mixture of (S,S)-4,5-bis-(4dihydroxyboranyl-phenyl)-2,2-dimethyl-[1,3]dioxolane $(S, S)-10 \quad(2.00 \mathrm{~g}, \quad 5.85 \mathrm{mmol})$, $\mathrm{Pd}\left(\mathrm{PPh}_{3}\right)_{4}(676 \mathrm{mg}, 0.58 \mathrm{mmol})$ and $\mathrm{Na}_{2} \mathrm{CO}_{3}(6.2 \mathrm{~g}, 58.5 \mathrm{mmol})$ in mixture of toluene (58 $\mathrm{ml})$, ethanol $(5.8 \mathrm{ml})$ and water $(3 \mathrm{ml})$. The reaction mixture was heated at $120^{\circ} \mathrm{C}$ for $24 \mathrm{~h}$. After completion of the reaction, the mixture was quenched with aqueous $\mathrm{NaOH}$ solution and extracted with dichloromethane. The organic extracts were combined and washed with brine, dried on anhydrous $\mathrm{MgSO}_{4}$ and filtered. The filtrate was then concentrated under reduced pressure to give a solid crude product. The residue was purified by column chromatography $\left(\mathrm{SiO}_{2}\right.$, with eluent of hexane : ethyl acetate $\left.=1: 3\right)$ to provide the product as a white solid (1.96 g, $82 \%) . \quad \mathrm{R}_{\mathrm{f}} 0.3$ (Hex : EA = $\left.1: 3\right)$; m.p. $=257 \sim 259{ }^{\circ} \mathrm{C} ;{ }^{1} \mathrm{H} \mathrm{NMR}\left(\mathrm{CDCl}_{3}, 300 \mathrm{MHz}\right): \delta$ $(\mathrm{ppm})=1.72(\mathrm{~s}, 6 \mathrm{H}), 4.82(\mathrm{~s}, 2 \mathrm{H}), 7.38(\mathrm{~d}, 4 \mathrm{H}, \mathrm{J}=7.9 \mathrm{~Hz}), 7.51(\mathrm{~d}, 4 \mathrm{H}, \mathrm{J}=5.8 \mathrm{~Hz}), 7.63(\mathrm{~d}$, $4 \mathrm{H}, \mathrm{J}=8.3 \mathrm{~Hz}), 8.66(\mathrm{~d}, 4 \mathrm{H}, \mathrm{J}=5.8 \mathrm{~Hz}) ;{ }^{13} \mathrm{C} \mathrm{NMR}\left(\mathrm{CDCl}_{3}, 75 \mathrm{MHz}\right): \delta(\mathrm{ppm})=27.41$, $85.26,110.11,121.75,127.36,127.69,137.91,138.38,147.98,150.51$; FT-IR $\left(\mathrm{KBr}, \mathrm{cm}^{-1}\right)$ 3027, 2882, 1592; MS (FAB, M+H): Calcd. for $\mathrm{C}_{27} \mathrm{H}_{24} \mathrm{~N}_{2} \mathrm{O}_{2}: 408.18$ found 409.1915; $[\alpha]_{\mathrm{D}}{ }^{20}=$ $-1170^{\circ}\left(\mathrm{C}=0.0205 \mathrm{~g} / \mathrm{L} \mathrm{CH}_{3} \mathrm{OH}\right)$.

Ethylene diamine palladium nitrate. $73.6 \mathrm{mg}(0.31 \mathrm{mmol})$ of ethylene diamine palladium chloride was suspended in $20 \mathrm{ml}$ water at RT. $\operatorname{AgNO}_{3}(105.3 \mathrm{mg} ; 0.62 \mathrm{mmol})$ was added to the suspension and the mixture stirred for $3 \mathrm{~h}$. A white solid of $\mathrm{AgCl}$ formed was filtered off. The filtrate contained ethylene diamine palladium nitrate which was diluted to double volume with methanol and used for the next step without further purification.

Ethylene diamine platinum nitrate. $60 \mathrm{mg}(0.18 \mathrm{mmol})$ of ethylene diamine platinum chloride was suspended in $20 \mathrm{ml}$ water at RT. $\mathrm{AgNO}_{3}(64 \mathrm{mg} ; 0.38 \mathrm{mmol})$ was added to the suspension and the mixture stirred for $30 \mathrm{~min}$. A white solid of $\mathrm{AgCl}$ formed and was filtered off. The filtrate contained ethylene diamine platinum nitrate and was diluted to double volume with methanol, which was used for the next step without further purification. 
General Procedure for the preparation of the rhombs. The rhombs were prepared according to the procedures given by Fujita et al. ${ }^{1}$ The ligand $(0.31 \mathrm{mmol})$ was dissolved in $\mathrm{MeOH}$ (40 $\mathrm{ml}$ ). The ethylenediamine palladium or platinum nitrate solution prepared previously was added to the solution. After stirring for $15 \mathrm{~h}$ at $0^{\circ} \mathrm{C}$, a small amount of insoluble material formed during the reaction was removed through filtration. The filtrate was dried under reduced pressure and the white solid obtained was again dissolved in $\mathrm{MeOH}$. The insoluble materials were filtered off again. The white solid product obtained by evaporating of the $\mathrm{MeOH}$ under reduced pressure was homogeneous enough for the subsequent analysis.

Rhomb (S,S,S,S)-1a. Yield: $140 \mathrm{mg}(65.7 \%) .{ }^{1} \mathrm{H}$ NMR ([D $\left.]-\mathrm{DMSO}, 300 \mathrm{MHz}\right): \delta(\mathrm{ppm})=$ 2.66(s, 8H), 3.22(s, 12H), 4.60(s, 4H), 5.64(s, 8H), 7.13 (d, 8H, J = 8.0 Hz), 7.68 (d, 8H, J = $8.2 \mathrm{~Hz}), 7.92(\mathrm{~d}, 8 \mathrm{H}, \mathrm{J}=6.3 \mathrm{~Hz}), 8.65(\mathrm{~d}, 8 \mathrm{H}, \mathrm{J}=6.3 \mathrm{~Hz}) ;{ }^{13} \mathrm{C}$ NMR ([D $\left.]-\mathrm{DMSO}, 75 \mathrm{MHz}\right)$ : $\delta(\mathrm{ppm})=46.78,56.71,85.11,123.26,126.56,129.04,133.91,140.69,150.01,151.87$; FT-IR $\left(\mathrm{KBr}, \mathrm{cm}^{-1}\right)$ : 3455, 3217, 3096, 1614, 1367; Calcd. for $\mathrm{C}_{56} \mathrm{H}_{64} \mathrm{~N}_{12} \mathrm{O}_{16} \mathrm{Pd}_{2}$ : 1372.2633; MS (ESI): $\mathrm{m} / \mathrm{z}=624.5\left\{\left[\mathrm{M}-2\left(\mathrm{NO}_{3}{ }^{-}\right)\right]^{2+}\right\} ;[\alpha]_{\mathrm{D}}{ }^{17}=-700^{\circ}(\mathrm{C}=0.0025 \mathrm{~g} / \mathrm{ml}, \mathrm{MeOH})$

Rhomb $(R, R, R, R)-1 a$. Yield $110 \mathrm{mg}(0.74 \mathrm{mmol} ; 65 \%)$. Spectral data identical with those of $(S, S, S, S)-1 \mathbf{a}$ except for $[\alpha]_{\mathrm{D}}{ }^{17}=+701^{\circ}(\mathrm{C}=0.0025 \mathrm{~g} / \mathrm{ml}, \mathrm{MeOH})$.

Rhomb (S,S,S,S)-1b Yield: $134.2 \mathrm{mg}(62 \%) .{ }^{1} \mathrm{H}$ NMR ([D $]$-DMSO, $\left.300 \mathrm{MHz}\right): \delta(\mathrm{ppm})=$ $1.63\left(\mathrm{~s}, 12 \mathrm{H}, 4 \mathrm{CH}_{3}\right), 2.70\left(\mathrm{~s}, 8 \mathrm{H}, 2 \mathrm{C}_{2} \mathrm{H}_{4}\right), 4.83(\mathrm{~s}, 4 \mathrm{H}, 4 \mathrm{CH}), 5.71\left(\mathrm{~s}, 8 \mathrm{H}, 4 \mathrm{NH}_{2}\right), 7.31$ and 7.34 $(\mathrm{d}, 8 \mathrm{H}, \mathrm{J}=8.2 \mathrm{~Hz}, \mathrm{Py}), 7.83$ and $7.85(\mathrm{~d}, 8 \mathrm{H}, \mathrm{J}=8.2 \mathrm{~Hz}, \mathrm{Py}), 7.96$ and 7.98 (d, 8H, J = 6.6 $\mathrm{Hz}, \mathrm{Ph}), 8.68$ and 8.70 (d, 8H, J = 6.0 Hz, Ph); FT-IR (KBr, $\mathrm{cm}^{-1}$ ): 3427, 3207, 3093, 2974, 1614, 1380, 1218, 1056, 817; Calcd. for $\mathrm{C}_{58} \mathrm{H}_{64} \mathrm{~N}_{12} \mathrm{O}_{16} \mathrm{Pd}_{2}$ : 1398.04; MS (ESI): $\mathrm{m} / \mathrm{z}=650.7$ $\left\{\left[\mathrm{M}-2\left(\mathrm{NO}_{3}{ }^{-}\right)-2\left(\mathrm{HNO}_{3}\right)-2\left(\mathrm{H}_{2}\right)\right]^{2+}\right\}, 713.7\left\{\left[\mathrm{M}-2\left(\mathrm{NO}_{3}{ }^{-}\right)\right]^{2+}\right\}$

Rhomb $(R, R, R, R)-\mathbf{1 b}$. Spectral data identical with those of $(S, S, S, S)-\mathbf{1 b}$.

Rhomb (S,S,S,S)-2a. Yield: $115.5 \mathrm{mg}(48 \%) .{ }^{1} \mathrm{H}$ NMR ([D $\left.]-\mathrm{MeOH}, 300 \mathrm{MHz}\right): \delta(\mathrm{ppm})=$ 2.79(s, $\left.8 \mathrm{H}, 2 \mathrm{C}_{2} \mathrm{H}_{4}\right), 3.23\left(\mathrm{~s}, 12 \mathrm{H}, 4 \mathrm{CH}_{3}\right), 4.50(\mathrm{~s}, 4 \mathrm{H}, 4 \mathrm{CH}), 7.13$ and $7.16(\mathrm{~d}, 8 \mathrm{H}, \mathrm{J}=8.3 \mathrm{~Hz}$, Py), 7.54 and 7.57 (d, 8H, $\mathrm{J}=8.2 \mathrm{~Hz}, \mathrm{Py}), 7.74$ and $7.78(\mathrm{~d}, 8 \mathrm{H}, \mathrm{J}=6.6 \mathrm{~Hz}, \mathrm{Ph}), 8.67$ and $8.69(\mathrm{~d}, 8 \mathrm{H}, \mathrm{J}=6.6 \mathrm{~Hz}, \mathrm{Ph}) ;{ }^{13} \mathrm{C}$ NMR $\left(\left[\mathrm{D}_{6}\right]-\mathrm{DMSO}, 75 \mathrm{MHz}\right): \delta(\mathrm{ppm})=46.97,56.75$,

\footnotetext{
${ }^{1}$ Fujita, M.; Yazaki, J.; Ogura, K. J. Am. Chem. Soc. 1990, 112, 5645-5647.
} 
85.01, 123.71, 126.52, 129.09, 133.72, 140.77, 149.80, 152.50; FT-IR (KBr, $\left.\mathrm{cm}^{-1}\right)$ : 3425, 3195, 3060, 2937, 1618, 1384, 1076, 819; Calcd. for $\mathrm{C}_{56} \mathrm{H}_{64} \mathrm{~N}_{12} \mathrm{O}_{16} \mathrm{Pt}_{2}$ : 1551.33; MS (ESI): $\mathrm{m} / \mathrm{z}=636.2\left\{\left[\mathrm{M}-2\left(\mathrm{NO}_{3}{ }^{-}\right)\right]^{2+}\right\}$

Rhomb (S,S,S,S)-2b. Yield: $135.2 \mathrm{mg}(55.4 \%) .{ }^{1} \mathrm{H}$ NMR ([D $\left.]-\mathrm{MeOH}, 300 \mathrm{MHz}\right): \delta$ (ppm) $=1.68\left(\mathrm{~s}, 12 \mathrm{H}, 4 \mathrm{CH}_{3}\right), 2.81\left(\mathrm{~s}, 8 \mathrm{H}, 2 \mathrm{C}_{2} \mathrm{H}_{4}\right), 4.78(\mathrm{~s}, 4 \mathrm{H}, 4 \mathrm{CH}), 7.33$ and $7.36(\mathrm{~d}, 8 \mathrm{H}, \mathrm{J}=8.0 \mathrm{~Hz}$, Py), 7.73 and 7.76 (d, 8H, J = 8.3 Hz, Py), 7.84 and $7.86(\mathrm{~d}, 8 \mathrm{H}, \mathrm{J}=6.5 \mathrm{~Hz}, \mathrm{Ph}), 8.72$ and $8.75(\mathrm{~d}, 8 \mathrm{H}, \mathrm{J}=6.5 \mathrm{~Hz}, \mathrm{Ph})$; FT-IR $\left(\mathrm{KBr}, \mathrm{cm}^{-1}\right): 3427,3197,3089,2918,1618,1380,1218$, 1049, 821; Calcd. for $\mathrm{C}_{58} \mathrm{H}_{64} \mathrm{~N}_{12} \mathrm{O}_{16} \mathrm{Pt}_{2}$ : 1575.36; MS (ESI): $\mathrm{m} / \mathrm{z}=441.8\left\{\left[\mathrm{M}-3\left(\mathrm{NO}_{3}{ }^{-}\right)^{-}\right.\right.$ $\left.\left.\left(\mathrm{HNO}_{3}\right)\right]^{3+}\right\}, 662.2\left\{\left[\mathrm{M}-2\left(\mathrm{NO}_{3}{ }^{-}\right)-2\left(\mathrm{HNO}_{3}\right)\right]^{2+}\right\}$ 


\section{Selected ${ }^{1} \mathbf{H}$ and ${ }^{13} \mathrm{C}$ NMR spectra}

a) Trans-4,4'-Dibromostilbene 4: ${ }^{1} \mathrm{H}$-NMR $\left(\mathrm{CDCl}_{3}\right)$

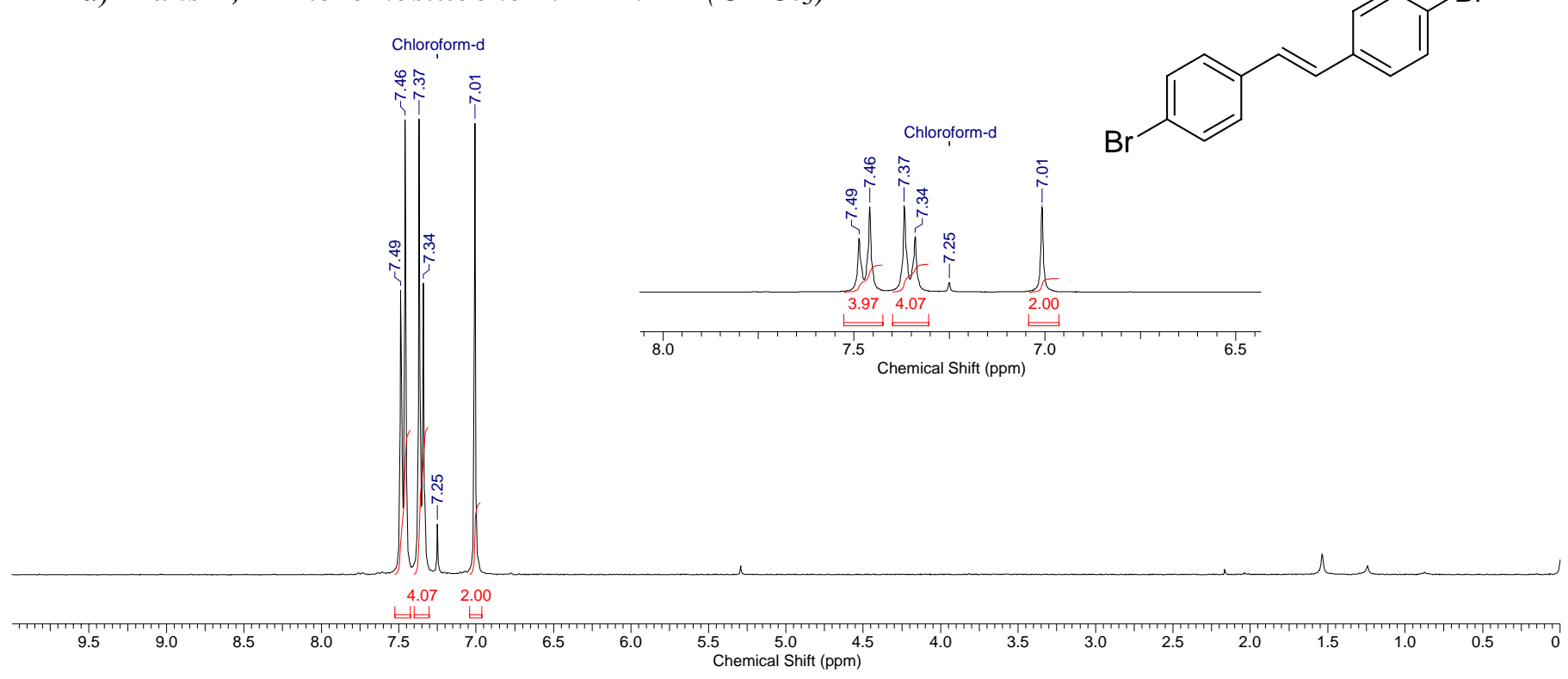

b) Trans-4,4'-Dibromostilbene $4:{ }^{13} \mathrm{C}-\mathrm{NMR}\left(\mathrm{CDCl}_{3}\right)$

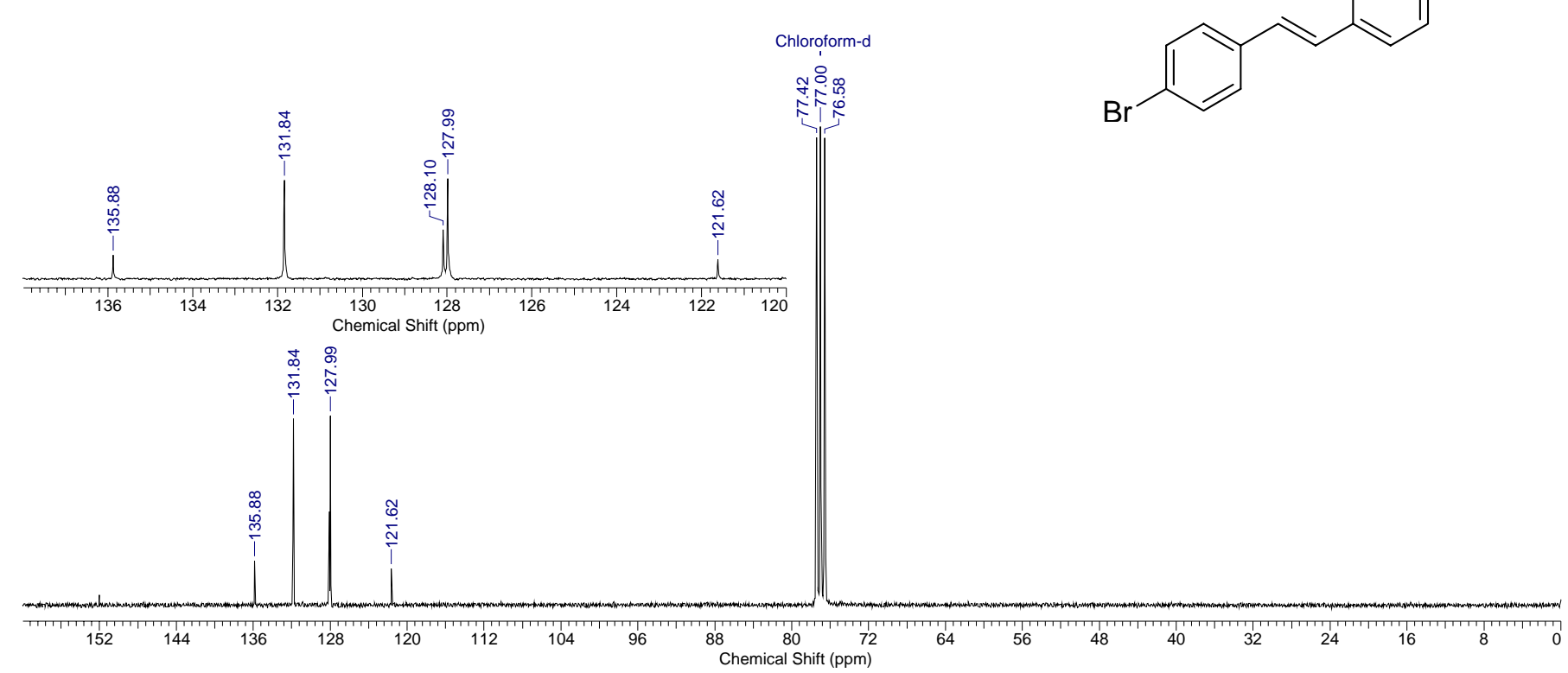


c) (S,S)-1,2-Bis(4-bromophenyl)-ethane-1,2-diol (S,S)-5: ${ }^{1} \mathrm{H}-\mathrm{NMR}\left(\mathrm{CDCl}_{3}\right)$

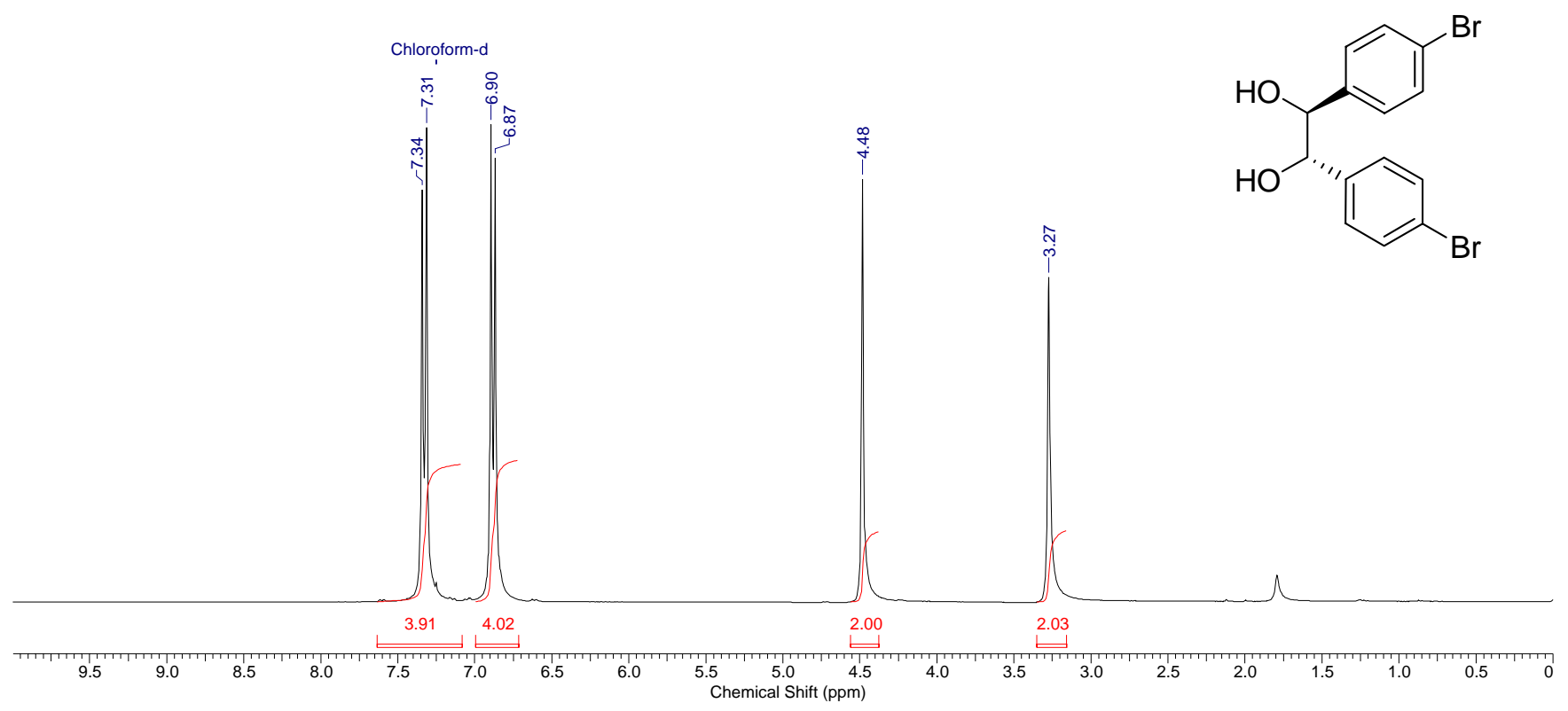

d) (S,S)-1,2-Bis(4-bromophenyl)-ethane-1,2-diol (S,S)-5: ${ }^{13} \mathrm{C}-\mathrm{NMR}\left(\mathrm{CDCl}_{3}\right)$

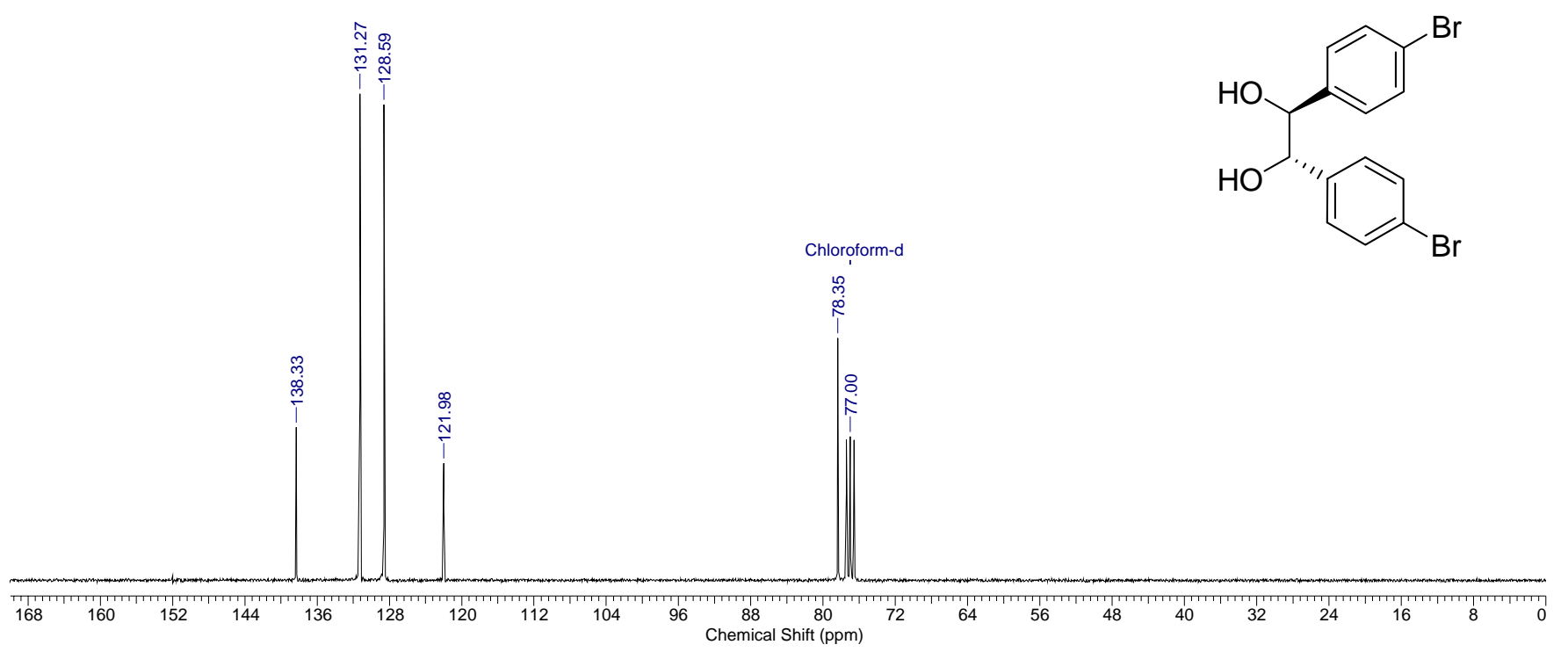


e) (S,S)-1,2-Bis(4-bromophenyl)-1,2-dimethoxy-ethane (S,S)-6: ${ }^{1} \mathrm{H}-\mathrm{NMR}\left(\mathrm{CDCl}_{3}\right)$

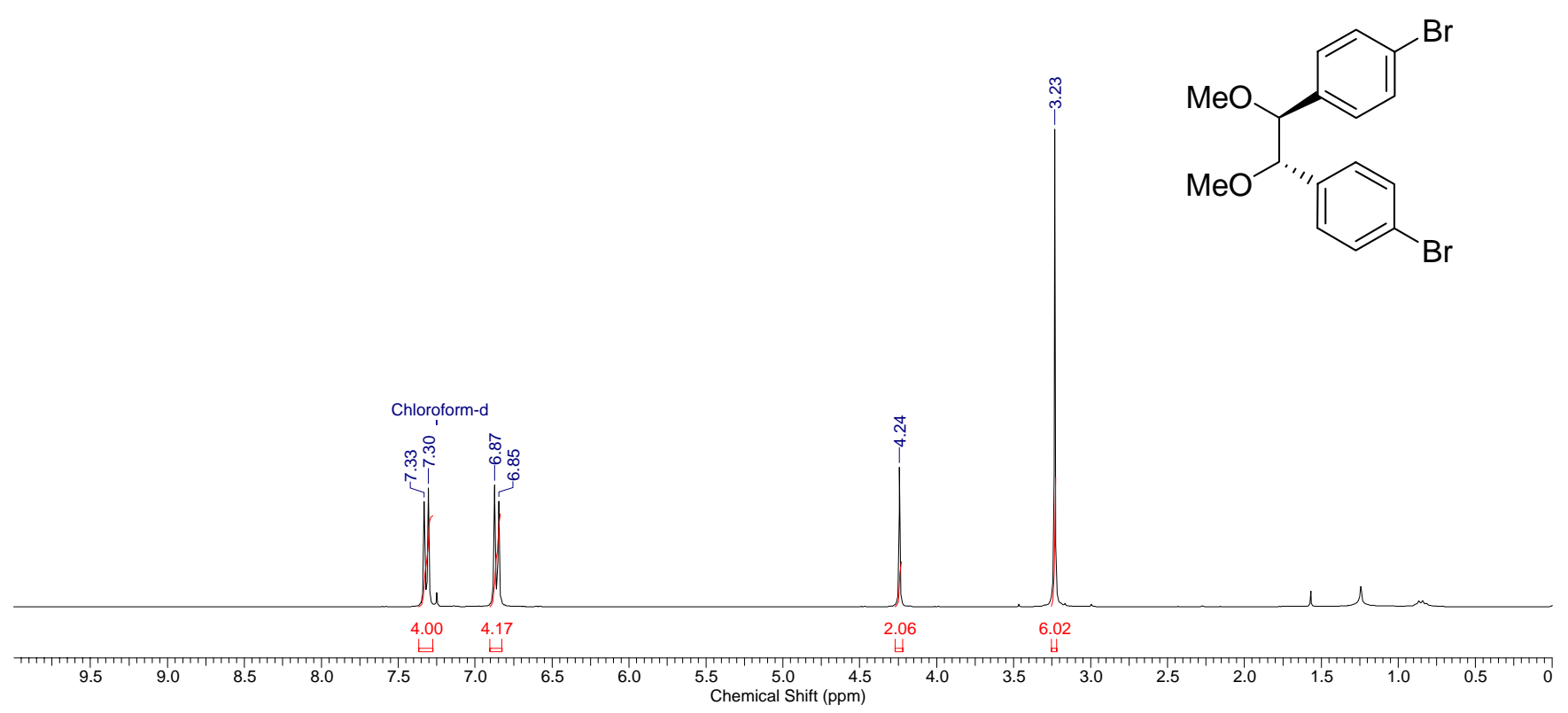

f) (S,S)-1,2-Bis(4-bromophenyl)-1,2-dimethoxy-ethane $(S, S)-6:{ }^{13} \mathrm{C}-\mathrm{NMR}\left(\mathrm{CDCl}_{3}\right)$

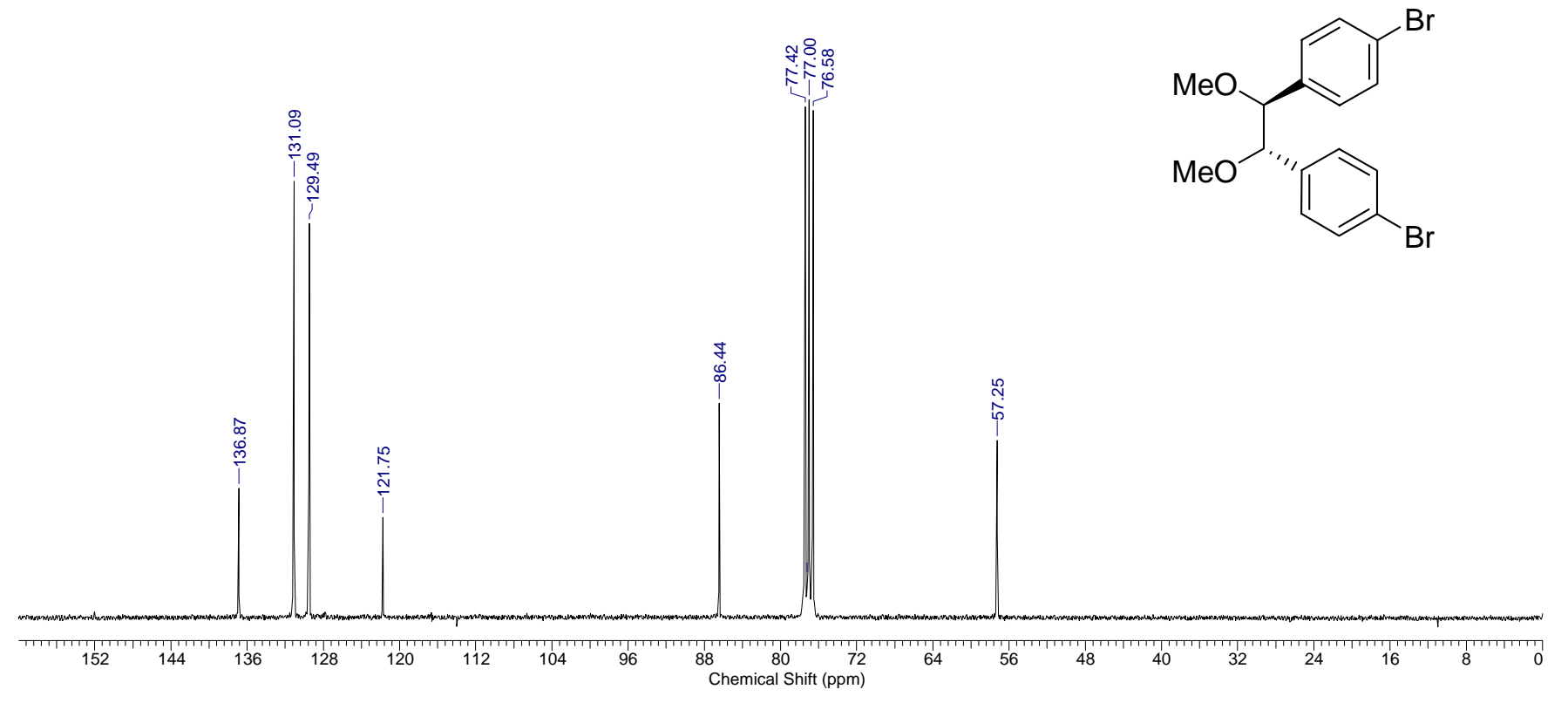


g) (S,S)-1,2-Bis(4-dihydroxyboranyl-phenyl)-1,2-dimethoxy-ethane (S,S)-7: ${ }^{1} \mathrm{H}-\mathrm{NMR}\left(C \mathrm{D}_{3} \mathrm{OD}\right)$

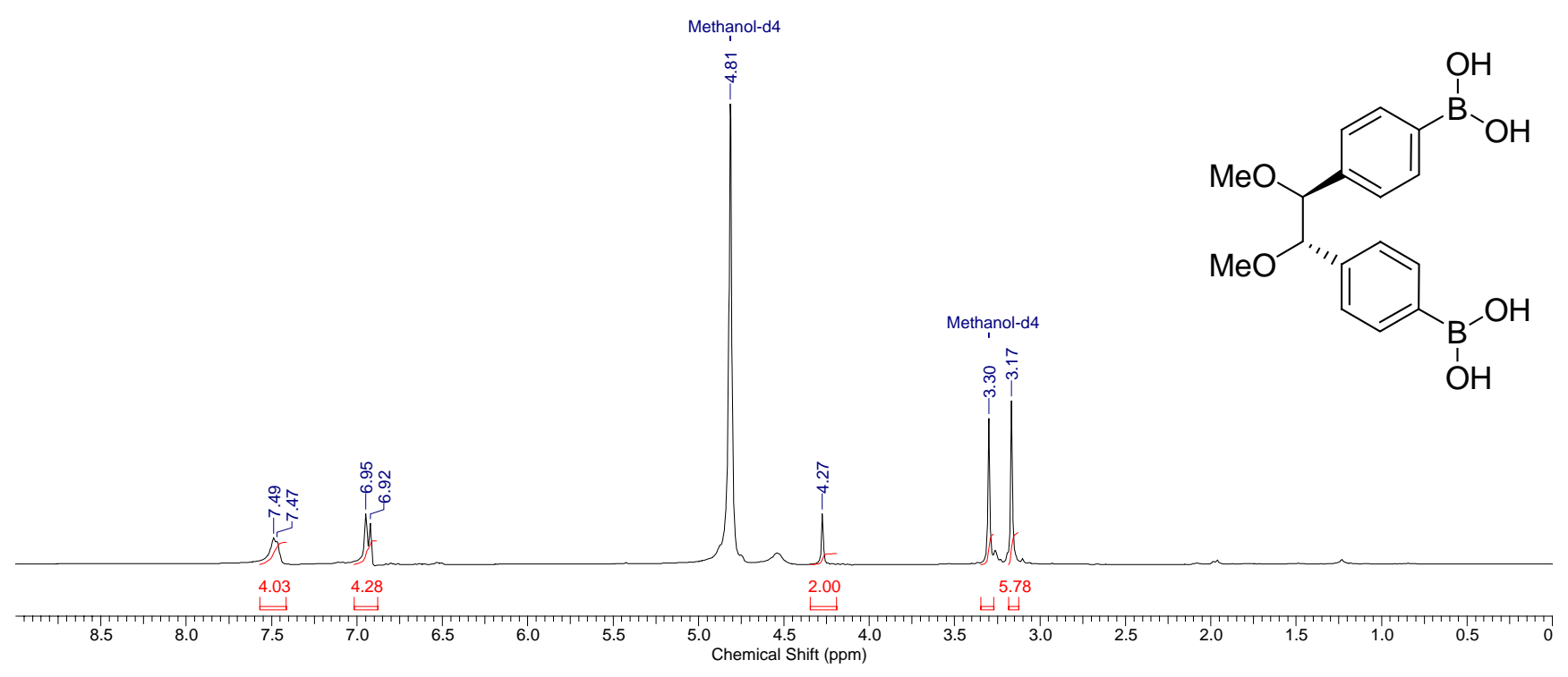

h) (S,S)-1,2-Bis(4-dihydroxyboranyl-phenyl)-1,2-dimethoxy-ethane (S,S)-7: ${ }^{13} C$-NMR (DMSO)<smiles>COC(c1ccc(B(O)O)cc1)c1ccc(B(O)O)cc1</smiles>
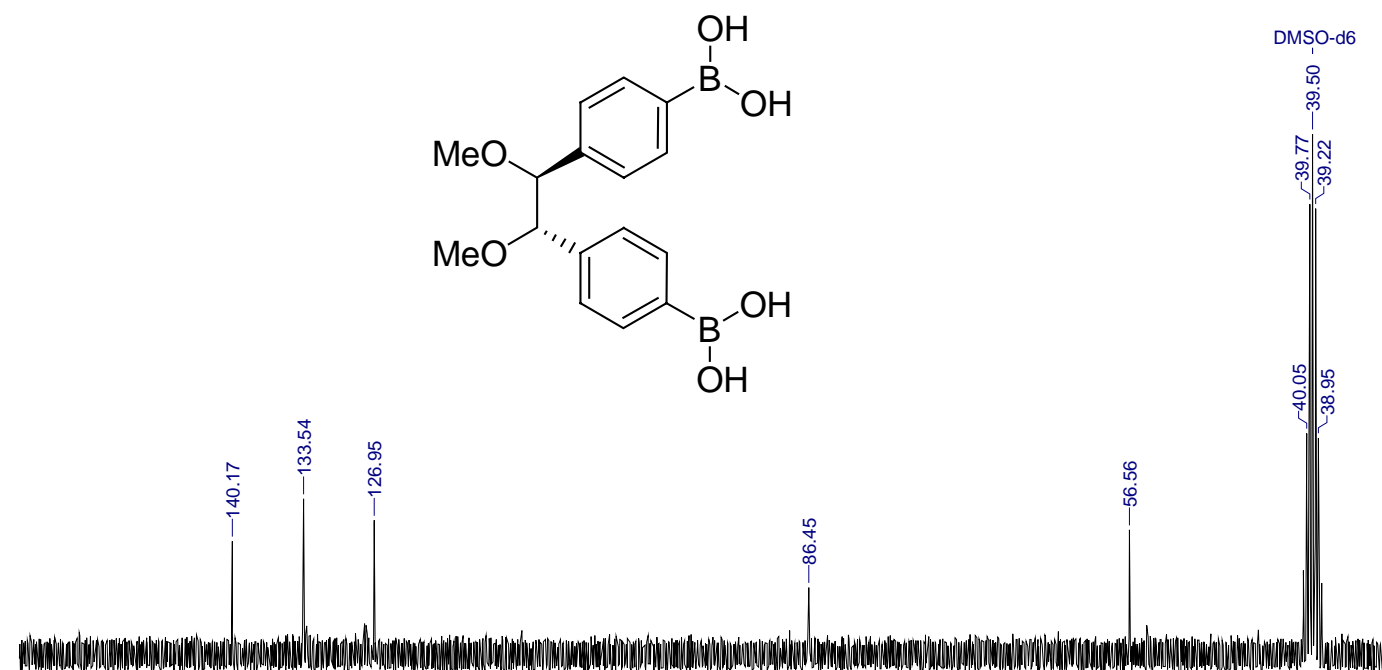

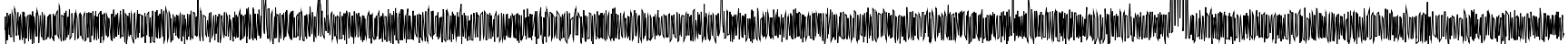

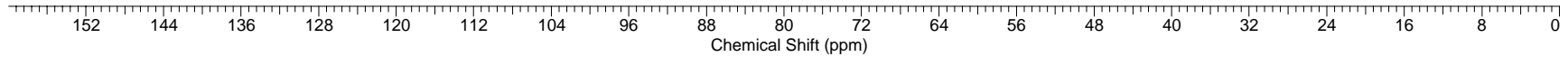


i) (S,S)-1,2-Bis(4-(4-pyridyl)-phenyl)-1,2-dimethoxy-ethane (S,S)-8: ${ }^{1} \mathrm{H}-\mathrm{NMR}\left(\mathrm{CDCl}_{3}\right)$

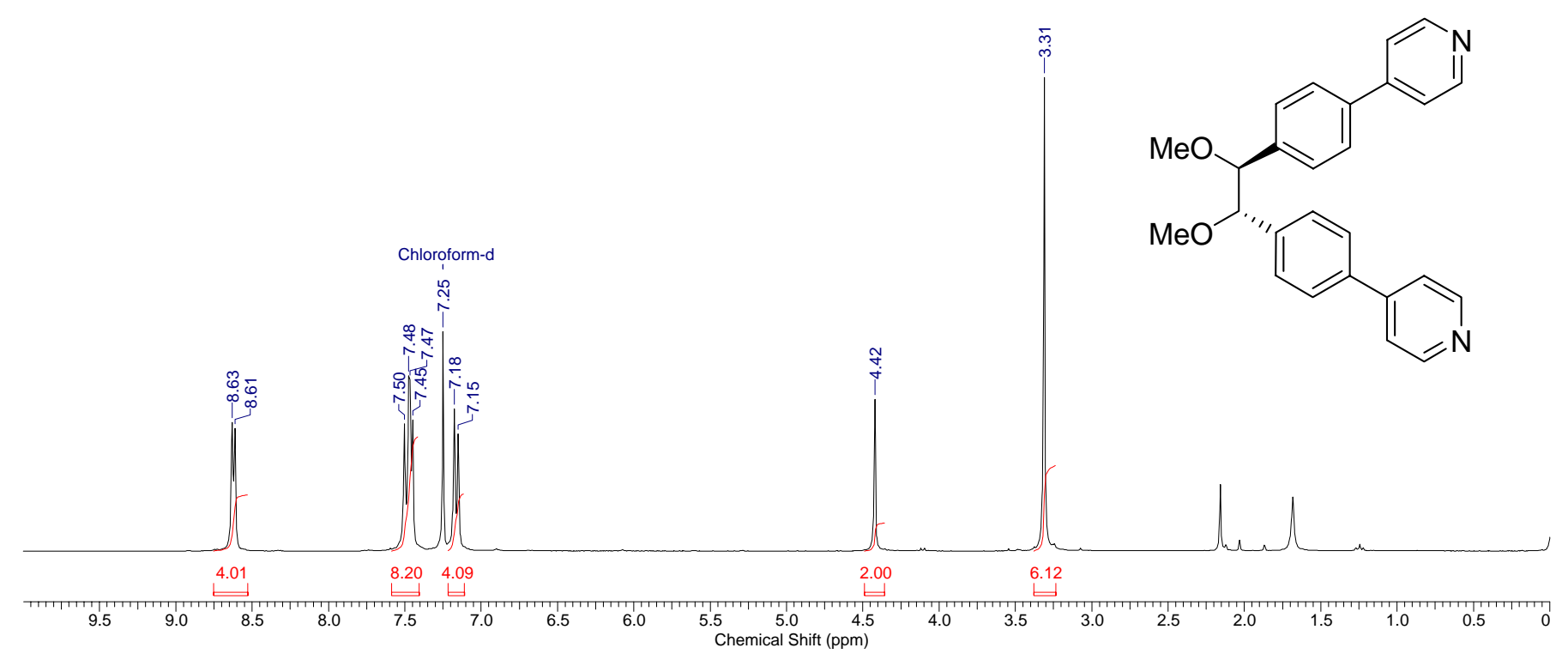

j) (S,S)-1,2-Bis(4-(4-pyridyl)-phenyl)-1,2-dimethoxy-ethane (S,S)-8: ${ }^{13} \mathrm{C}-\mathrm{NMR}\left(\mathrm{CDCl}_{3}\right)$

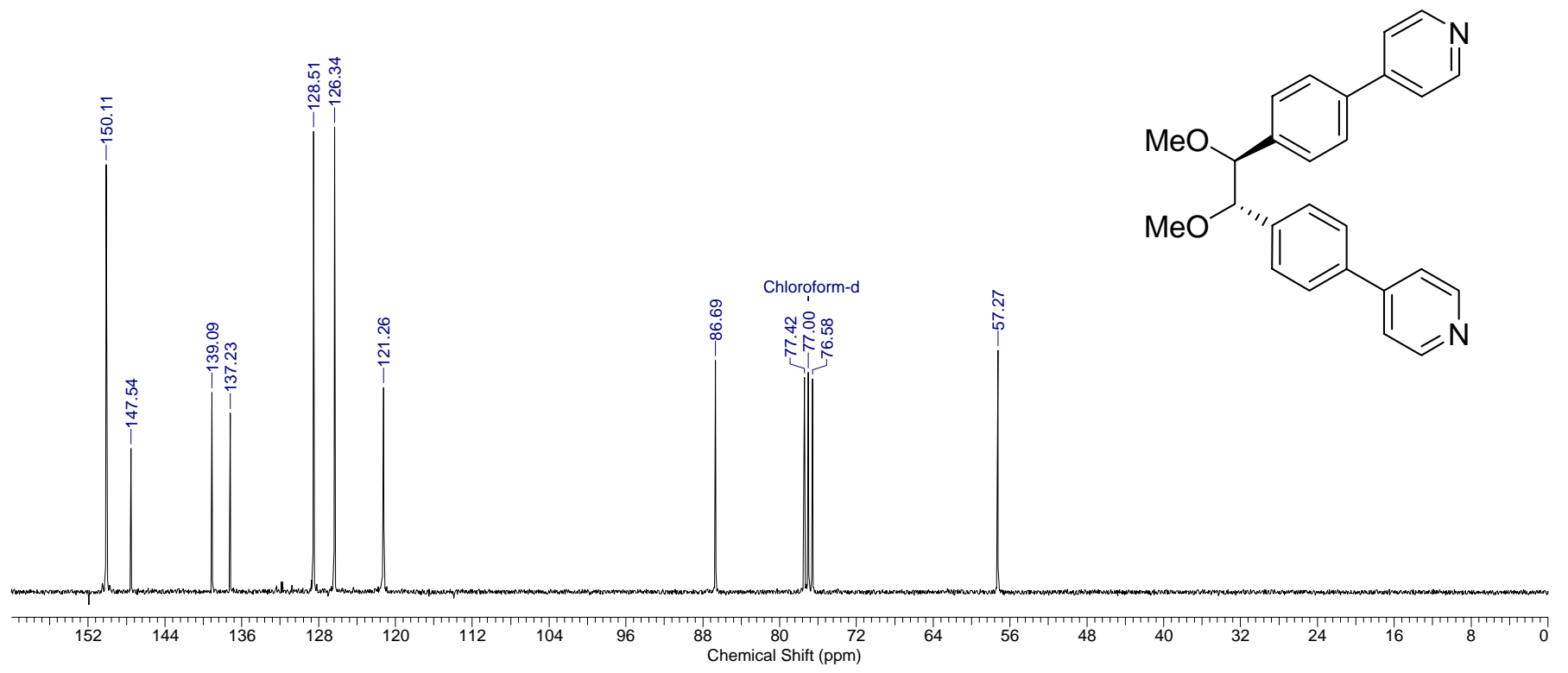


k) $\operatorname{Rhomb}(S, S, S, S)-1 a:{ }^{1} H-N M R(D M S O)$

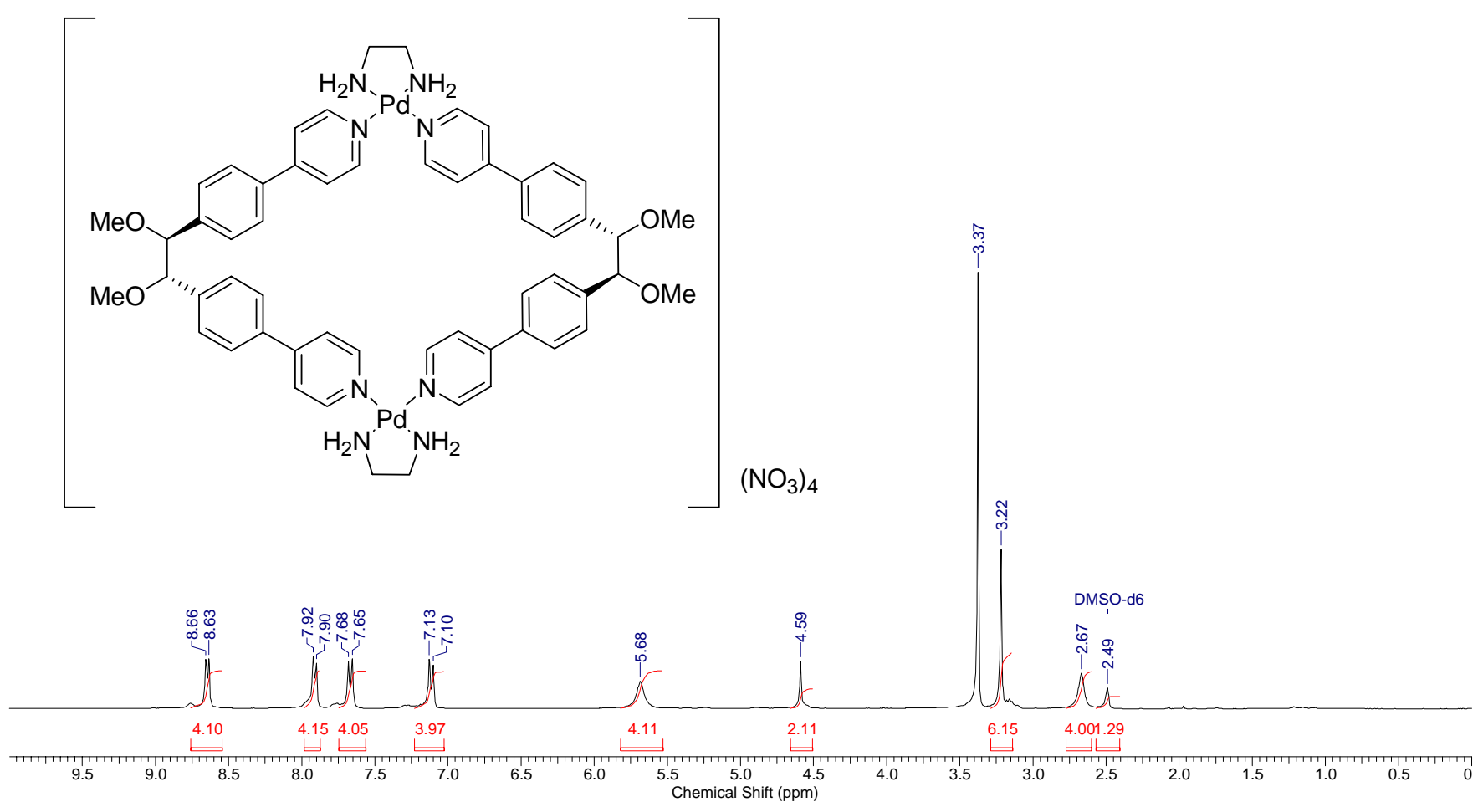

1) Rhomb (S,S,S,S)-1a: ${ }^{13} C-N M R$ (DMSO)

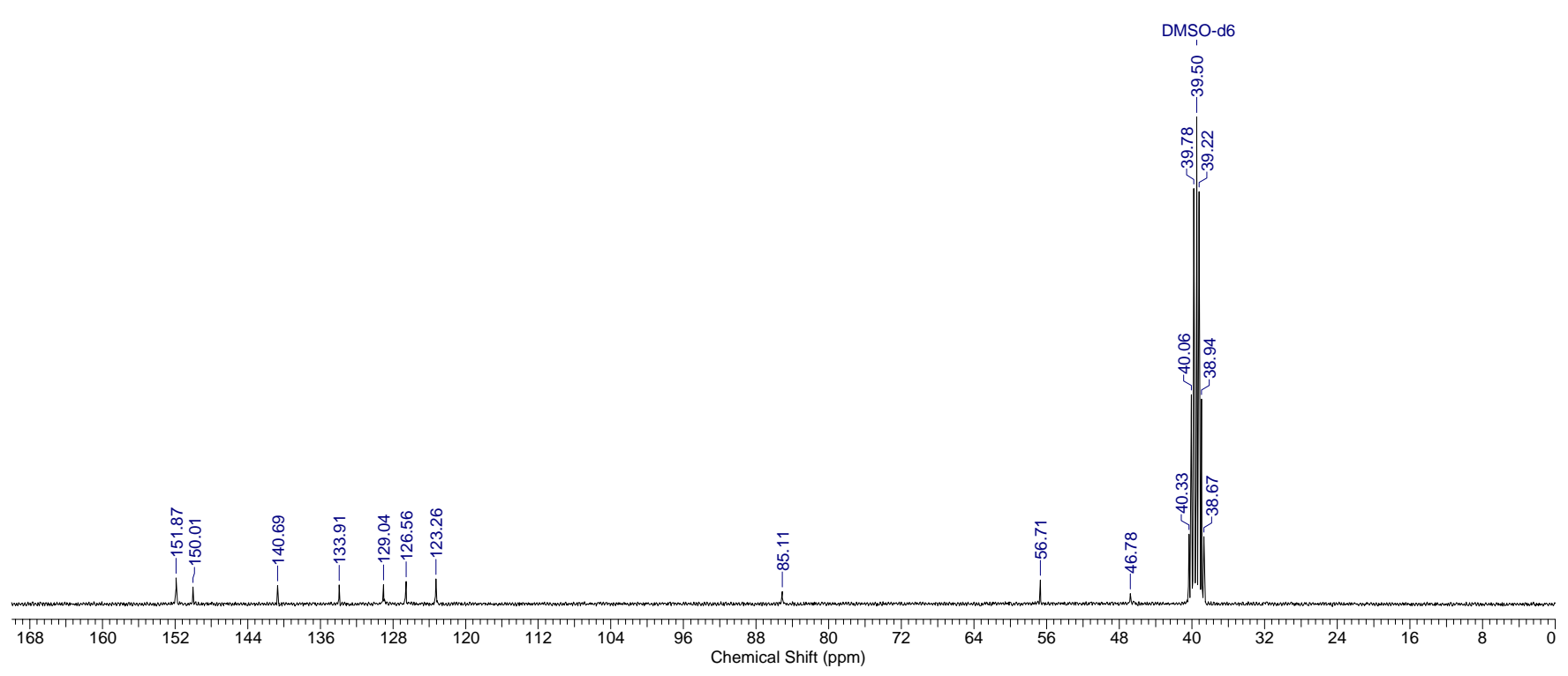


k) Rhomb $\left.(S, S, S, S)-\mathbf{1 b}:{ }^{1} H-N M R\left(C D_{3} O D / D_{2} O\right)\right)$

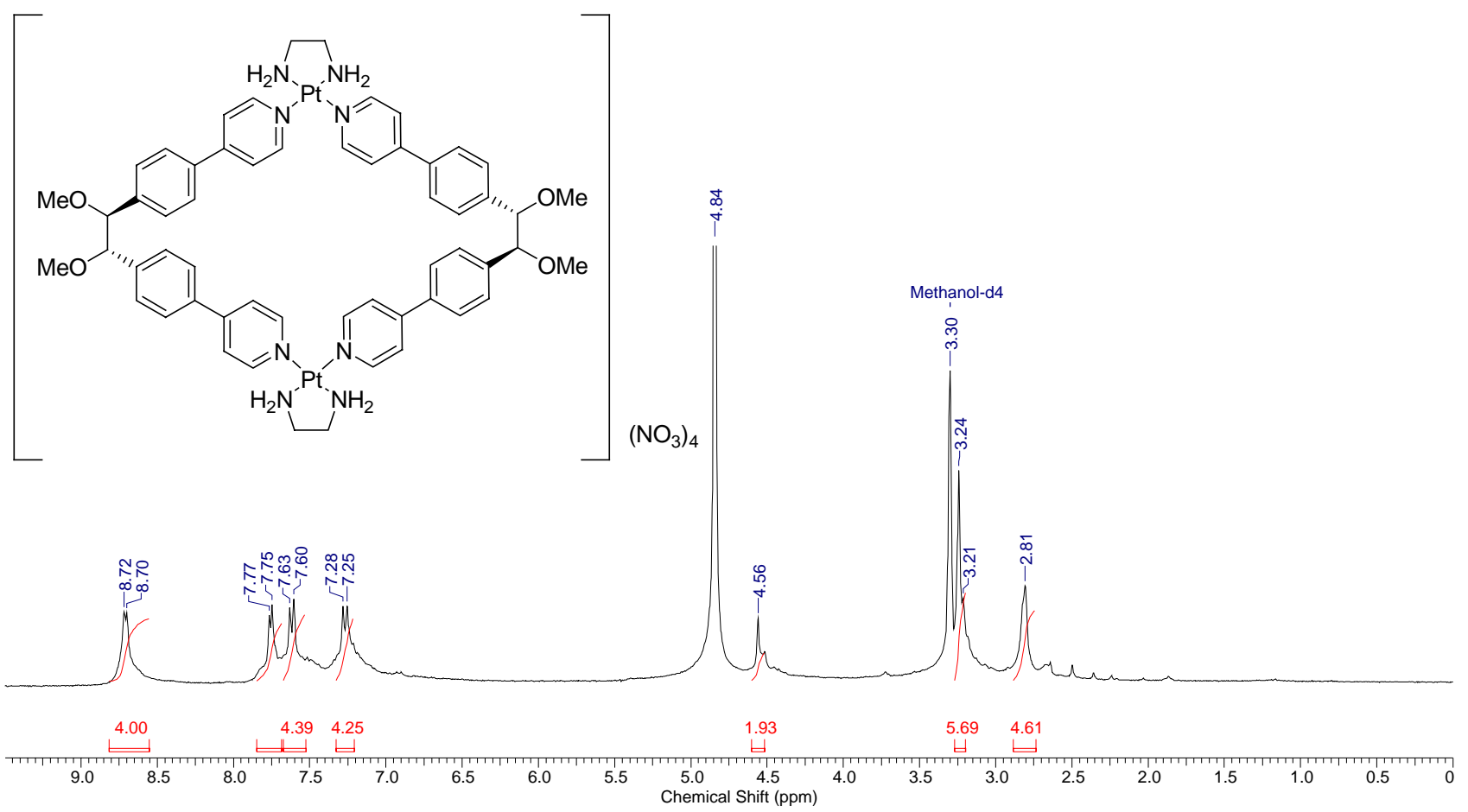




\section{Theoretical details for rhomb $2 b$}

Rhomb 2b was treated with the same DFT calculations as $\mathbf{1 b}$. We include here the structural details and the 14 highest occupied and 14 lowest unoccupied molecular orbitals which show that the features of this macrocycle are closely related to those of $\mathbf{1 b}$. The deviation from a square is somewhat more pronounced for $\mathbf{2} \mathbf{b}$ as compared to $\mathbf{1 b}$.
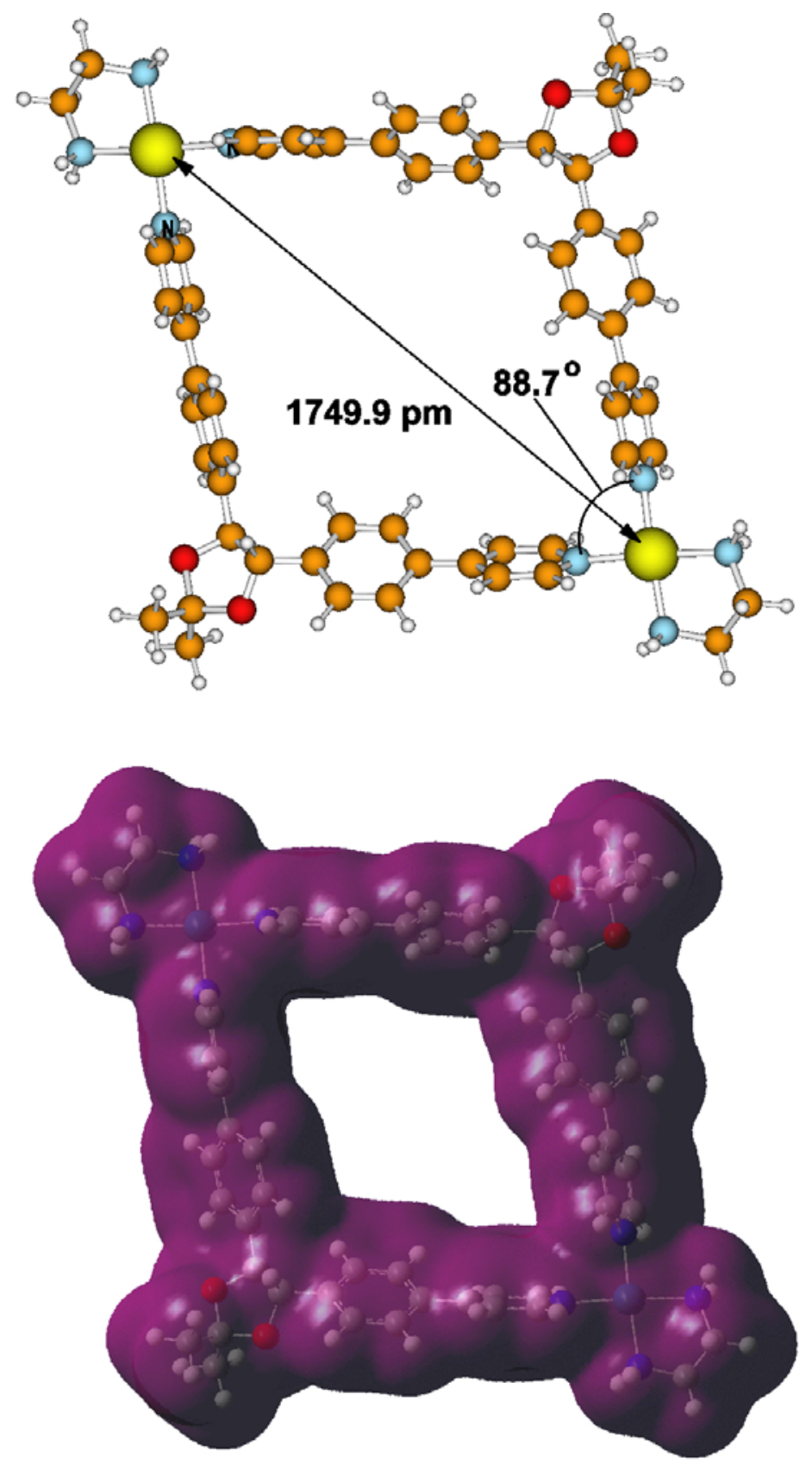

Figure S1. Optimized BP86/RI/TZVP structure of metallo-supramolecular macrocycle $\mathbf{2 b}$ (top). A plot of the electronic density, which nicely displays the acute angle at the two platinum corners and the obtuse angle at the acetal corners, is shown at the bottom. 


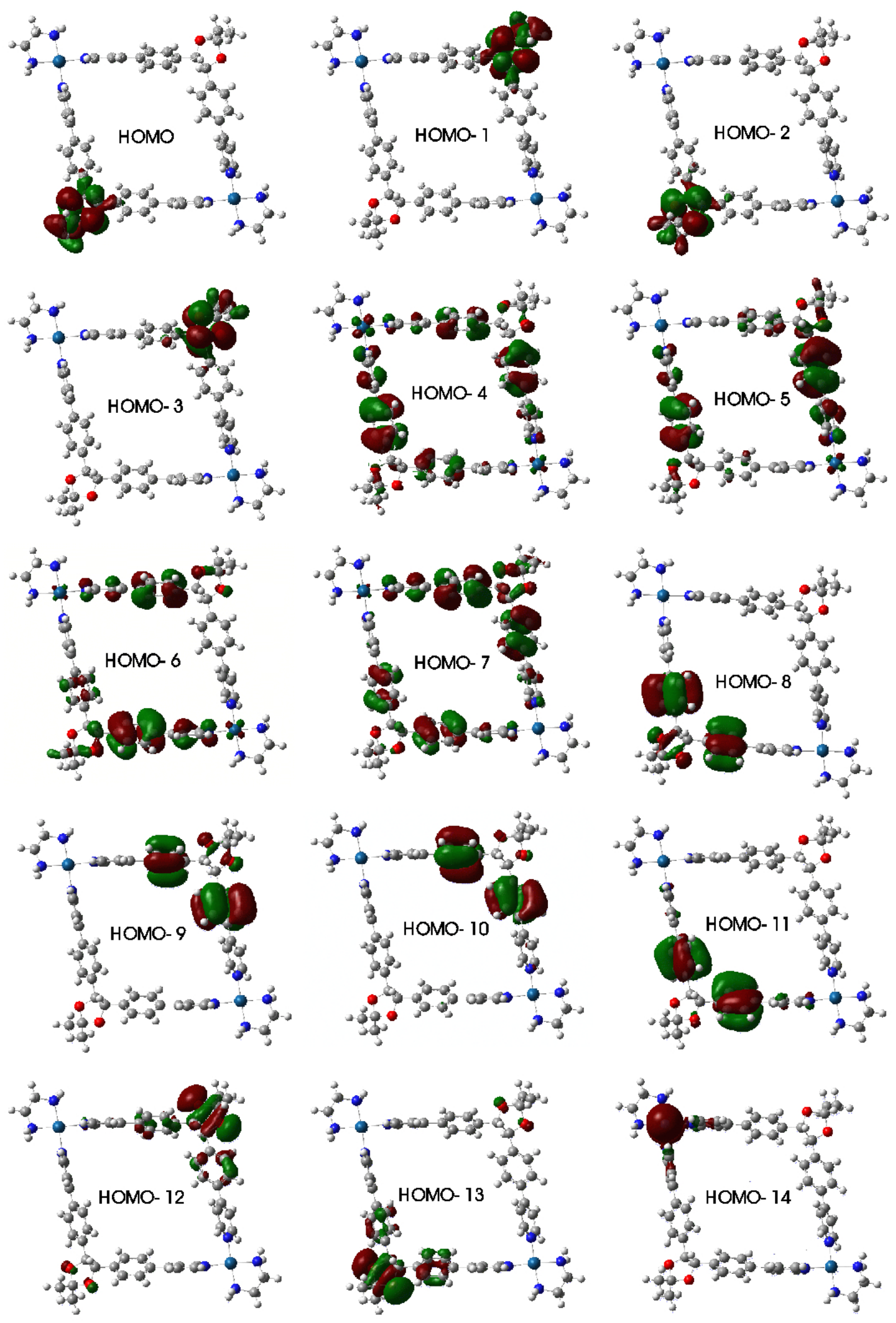

Figure S2. Highest occupied molecular orbitals of the macrocycle. Note that a dense "band" of frontier MOs exists, which solely consists of acetal-corner MOs. The first MO with metalcharacter AOs is the HOMO-14. For symmetry reasons near-degenerate pairs of orbitals exists. 


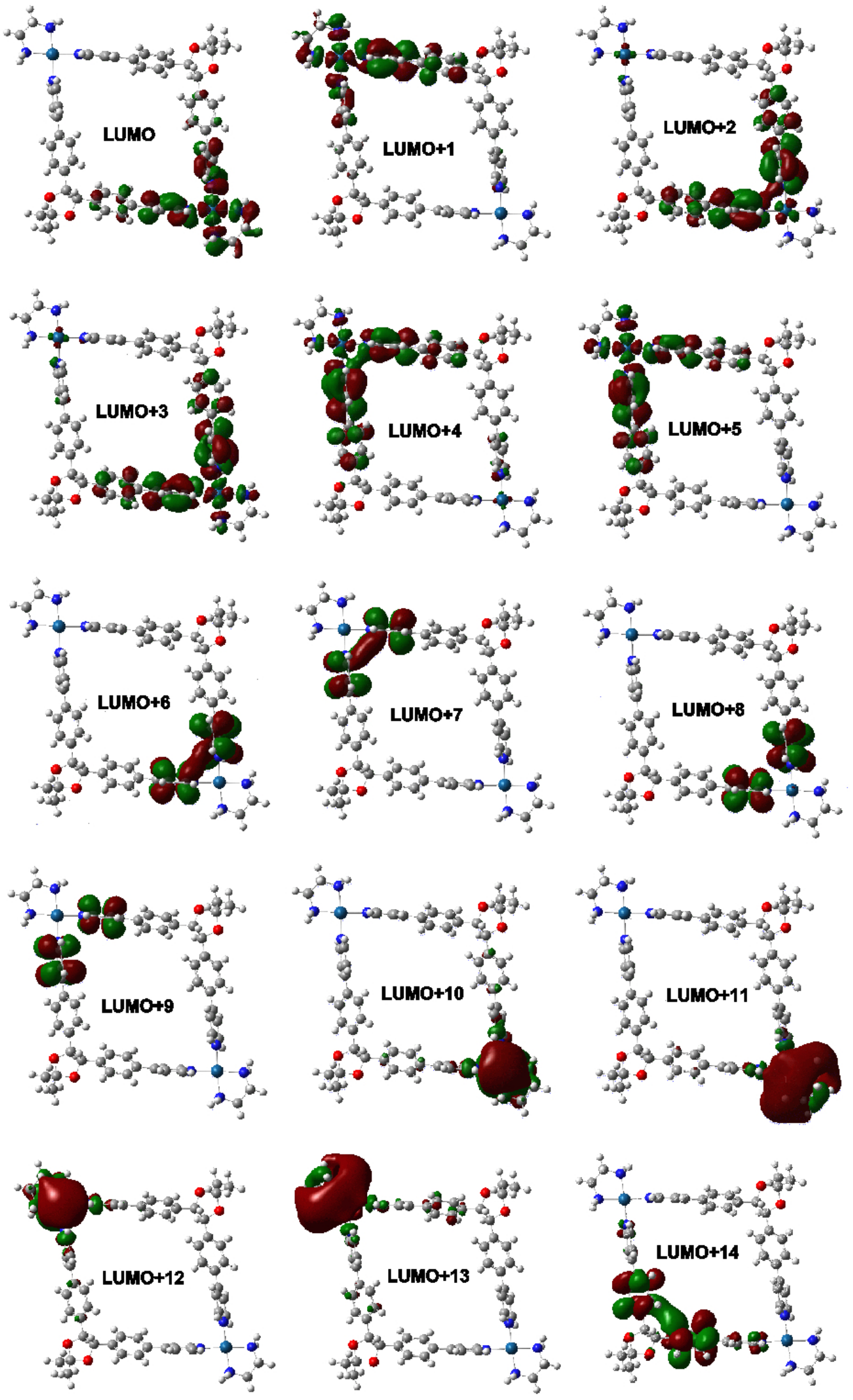

Figure S3. Low lying unoccupied molecular orbitals of the macrocycle. Note that a dense "band" of virtual frontier MOs exists, which solely consists of MOs centered at the metal corners. The first MO with AOs at the acetal corners is the LUMO+14. For symmetry reasons near-degenerate pairs of orbitals exists. 